\title{
Attempts to isolate Campylobacter jejuni from various body sites
}

\author{
EP WRIGHT
}

From the Public Health Laboratory, Luton and Dunstable Hospital, Lewsey Road, Luton LU4 ODZ

SUMMARY Clinical material collected from various body sites, but excluding faeces, was cultured on either selective or non-selective media for Campylobacter spp. Campylobacter jejuni was found in only two $(0 \cdot 18 \%)$ of 1100 specimens; both positive specimens were urine. From these results it is suggested that $C$ jejuni is an uncommon finding in clinical material other than faeces.

Campylobacter jejuni is now recognised as a leading cause of acute bacterial gastroenteritis in humans. Over 9500 cases were reported in Britain during $1980 .^{1}$ At present there are few reports of the isolation of $C$ jejuni from specimens other than faeces. Extraintestinal isolations have included blood, ${ }^{2}$ cerebrospinal fluid, ${ }^{3}$ bile,${ }^{4}$ urine,${ }^{5}$ cervix ${ }^{6}{ }^{6}$ vagina, ${ }^{7}$ joint fluid, ${ }^{8}$ appendix, ${ }^{9}$ and peritoneum. ${ }^{10}$ However the reports give few details of whether these isolates were incidental findings or found as part of a routine examination for $C$ jejuni in clinical specimens. The present study was undertaken to determine the frequency of $C$ jejuni isolation from samples taken from various body sites submitted for routine diagnostic bacteriology.

\section{Materials and methods}

Specimens of pus, tissue or fluid were collected from patients in Fazakerley Hospital, Liverpool, between October 1977 and August 1979 and from patients in Luton and Dunstable Hospital between November 1980 and September 1982. In addition cervical swabs were obtained from patients attending a Special Treatment Clinic in Liverpool during 1979 and semen was collected from men attending for infertility or post-vasectomy studies in Luton during 1981-2. These specimens were transported to the laboratory either in sterile bottles or on swabs. The swabs used were of absorbent cotton wool or Hospiswab (Medical Wire \& Equipment Co Ltd) both held in Stuart's transport medium at room temperature for up to $24 \mathrm{~h}$.

After appropriate routine culture each specimen was further inoculated onto blood agar if the specimen was taken from a site normally regarded as

Accepted for publication 19 January 1983 sterile or inoculated onto Skirrow's medium ${ }^{11}$ if taken from a site with normal flora. Throughout the investigation all plates were incubated at $43^{\circ} \mathrm{C}$ in an atmosphere of approximately $5 \%$ oxygen, $10 \%$ carbon dioxide and $85 \%$ hydrogen obtained by using an evacuation-replacement method in an anaerobic jar without a catalyst; cultures were examined after two days. Smears from suspect colonies checked for positive oxidase and catalase reactions, were identified as $C$ jejuni by their characteristic morphology on Gram staining. All campylobacter isolates were biotyped. ${ }^{12}$ During the period of study details were recorded of those patients who had campylobacter enteritis at the time the specimen was collected.

\section{Results}

The distribution of specimens from the various body sites examined is shown in the Table. Of 1100 specimens examined only two $(0 \cdot 18 \%)$ yielded $C$ jejuni; both these specimens were urine.

\section{CASE 1}

A seven-month old boy was admitted to hospital following a febrile convulsion associated with watery diarrhoea. Physical examination was unremarkable apart from a temperature of $40^{\circ} \mathrm{C}$. Stool cultures grew $C$ jejuni but no other enteropathogens. A urine specimen obtained by a collecting-bag showed no red or white cells on microscopy and no significant growth on cysteine lactose electrolyte-deficient (CLED) medium; however culture of the urine deposit on campylobacter-selective medium yielded $C$ jejuni. Both stool and urine isolates were $C$ jejuni biotype 1 . The child made an uneventful recovery with oral fluids and erythromycin. A month later he was symptom-free and stool and urine specimens 
Table 1 Specimens investigated for Campylobacter jejuni

\begin{tabular}{lll}
\hline Site & No. of specimens & Type of specimen (number) \\
\hline Respiratory & 317 & Pleural fluid (246) Throat (60) Sinus (11) \\
Male Genital & 243 & Semen (226) Hydrocoele (9) Scrotum (2) Urethra (4) Prostate (1) Epididymis (1) \\
Female Genital & 153 & Cervix (138) Vagina (5) Bartholin's abscess (4) Placenta (2) Uterus (1) \\
& & Liquor (1) Vulva (1) Products of conception (1) \\
Abdominal & 141 & Peritoneum (42) Bile (39) Gallstone (21) Gastric aspirate (17) Appendix (14) \\
& 77 & Lymph node (3) Pancreas (2) Liver (2) Duodenum (1) \\
Joints & 20 & Knee (58) Elbow (7) Unknown (6) Shoulder (3) Ankle (2) Hip (1) \\
Anorectal & 15 & Perianal (7) Buttock (5) Ischiorectal (5) Pilonidal (3) \\
Renal & 15 & Urine (10) Calculus (3) Kidney (2) \\
Skin & 119 & 'Abscess' (8) Breast (3) Axilla (3) Sebaceous cyst (1) \\
Miscellaneous & Cerebrospinal fluid (102) Breast milk (8) Bone (6) Ear (2) Eye (1) \\
\hline
\end{tabular}

were negative for $C$ jejuni. It is probable that the urine specimen was contaminated during collection by faecal material from the child's skin.

\section{CASE 2}

A 16-year-old boy was admitted to hospital following a camping holiday in France after which he developed bloody diarrhoea and abdominal pain. There was abdominal tenderness on examination. Stool cultures grew $C$ jejuni but no other enteropathogens. A mid-stream urine specimen contained no red or white cells and yielded a scanty mixed growth of doubtful significance on CLED medium. Culture of the urine deposit on campylobacter selective medium yielded $C$ jejuni. Both stool and urine isolates were $C$ jejuni biotype 1 . His symptoms quickly settled with intravenous fluids and erythromycin. At no time did he complain of symptoms referrable to the urinary tract. Two weeks later a stool specimen was negative for $C$ jejuni but unfortunately a further urine specimen was not examined. The possibility for faecal contamination of the urine must again be considered.

In addition to these two cases, six urines, two cerebrospinal fluids, one vaginal abscess swab and one knee aspirate were collected from 10 patients suffering from campylobacter enteritis at the time their specimen was collected.

\section{Discussion}

The results of this investigation suggest that $C$ jejuni is rarely found in clinical material other than faeces. This is consistent with the few case reports of extraintestinal isolations of $C$ jejuni of which most isolations have been from blood cultures; 40 cases of $C$ jejuni bacteraemia were collected in four years at one centre. ${ }^{2}$ The interpretation of previous reports of extraintestinal campylobacter isolations in the last 40 years is difficult because often the organisms were not fully identified and the taxonomy of the genus has until recently been poorly understood. Often the authors failed to appreciate the difference between the types of infection caused by the two campylobacter subspecies commonly associated with human infections; Campylobacter fetus ss fetus is a rare opportunist that causes invasive infection only in those with serious underlying disease whereas $C$ jejuni is a common and sometimes invasive enteropathogen of normal people.

There is an increasing recognition of the complications which can arise in association with $C$ jejuni infections such as reactive arthritis, cholecystitis and septic abortion; however in the present study it was not possible to collect detailed information about the clinical diagnosis of each patient studied.

The frequency of $C$ jejuni isolation from clinical material might be increased if more blood cultures were taken during the early stages of campylobacter enteritis and also if other clinical specimens were specially examined for $C$ jejuni using appropriate isolation techniques. In this study the use of enrichment may also have resulted in an increased isolation rate; however further work needs to be undertaken to determine the limit of detection of $C$ jejuni by direct culture and enrichment from clinical material similar to that examined in this investigation. Recently a new selective medium for $C$ jejuni ${ }^{13}$ has been successful in increasing the frequency of isolation from faeces. A study using this medium and enrichment broth for isolating $C$ jejuni from various body sites has recently commenced.

Part of the work in this study was undertaken at the Regional Public Health Laboratory, Fazakerley Hospital, Liverpool.

\footnotetext{
References

' PHLS Communicable Disease Surveillance Centre. Campylobacter infections, 1977-80. Br Med J 1981;282:1484.

2 Young SEJ. Bacteraemia 1975-1980: a survey of cases reported to the PHLS Communicable Disease Surveillance Centre. $J$ Infect 1982;5:19-26.

${ }^{3}$ Norrby R, McCloskey RV, Zackrisson G, Falsen E. Meningitis
} 
caused by Campylobacter fetus ssp. jejuni. Br Med J 1980;280: 1164 .

${ }^{4}$ Mertens A, De Smet M. Campylobacter cholecystitis. Lancet 1979;i:1092-3.

${ }^{5}$ Davies JS, Penfold JB. Campylobacter urinary infection. Lancet 1979;i:1091-2.

- Wright EP, Balsdon MJ, Okubadejo OA. Isolation of Campylobacter jejuni from cervix. Lancet 1982;ii:380.

' Anders BJ, Lauer BA, Paisley JW. Campylobacter gastroenteritis in neonates. Am J Dis Child 1981;135:900-2.

${ }^{8}$ Kowalec JK, Kaminski ZC, Krey PR. Campylobacter arthritis. Arthritis Rheum 1980;23:92-4.

9 Megraud F, Tachoire C, Latrille J, Bondonny JM. Appendicitis due to Campylobacter jejuni. Br Med J 1982;285:1165-6.

${ }^{10}$ Pearson AD, et al. Campylobacter infections in patients present- ing with diarrhoea, mesenteric adenitis and appendicitis. In: Newell DG, ed. Campylobacter: epidemiology, pathogenesis and biochemistry. Lancaster: MTP Press, 1982:147-51.

" Skirrow MB. Campylobacter enteritis: a 'new' disease. Br Med J 1977;ii:9-11.

12 Skirrow MB, Benjamin J. Differentiation of enteropathogenic campylobacter. J Clin Pathol 1980;33:1122.

${ }^{13}$ Bolton FJ, Robertson L. A selective medium for isolating Campylobacter jejuni/coli. J Clin Pathol 1982;35:462-7.

Requests for reprints to: Dr EP Wright, Public Health Laboratory, Luton \& Dunstable Hospital, Lewsey Road, Luton LU4 0DZ, England. 